\title{
Procesos administrativos en la gestión escolar del director de las instituciones educativas
}

\author{
Administrative processes in the school management of the director of \\ educational institutions
}

\section{DILIA ORTEGA}

diliaestherortega@gmail.com

Nuestra Señora del Tránsito Usiacuri, Colombia
- MAIGUALIDA BEJAS MONZANT

maigualidabejas@yahoo.com

Universidad del Zulia, Venezuela

Artículo recibido en septiembre 2018 / Arbitrado en octubre 2018 / Publicado en enero 2019

Resumen El propósito principal de esta investigación fue analizar los procesos administrativos en la gestión escolar del director de las instituciones educativas oficiales del Departamento Atlántico-Colombia. Se enmarcó como descriptiva, con un diseño de campo, transeccional y no experimental. La población estuvo constituida por ciento ocho sujetos específicamente cuatro directivos y ciento cuatro docentes siendo estos últimos una muestra control, que prestan sus servicios profesionales en los centros educativos De Sabana Larga y Nuestra señora del tránsito ambas ubicadas Departamento AtlánticoColombia. Para el análisis de los datos se empleó la estadística descriptiva. Se concluyó que en las instituciones educativas en contexto se detectaron debilidades por parte de los directivos, de acuerdo a la percepción de los docentes, en cada una de las dimensiones de las variables objetos de estudio.

Palabras clave: Procesos administrativos; gestión escolar; organizaciones educativas

Abstract The main purpose of this research was to analyze the administrative processes in the school management of the director of the official educational institutions of the Atlántico-Colombia Department. It was framed as descriptive, with a field design, transectional and not experimental. The population was made up of one hundred and eight subjects, specifically four managers and one hundred and four teachers, the latter being a control sample, who provide their professional services in the educational centers De Sabana Larga and Nuestra Señora del Transit, both located in the AtlánticoColombia Department. For the analysis of the data, descriptive statistics was used. It was concluded that in educational institutions in context, weaknesses were detected by the managers, according to the teachers' perception, in each of the dimensions of the variables under study.

Keywords: $\quad$ Administrative Processes; School Management; Educational Organizations 


\section{INTRODUCCIÓN}

En la actualidad la globalización, además de la alta competitividad en los mercados, ha obligado a las instituciones educativas a mirar hacia adentro y reconocer la importancia de su talento humano. Así pues, un ente educativo necesita de una estructura definida y de estrategias efectivas. El trabajo en equipo por parte de los miembros de la organización es vital para el desarrollo de las ideas que permitan el logro de los objetivos.

Dentro de este contexto, las organizaciones educativas de hoy en día, requieren mecanismos que alineen la estructura de las mismas, así como de normas y procedimientos, los cuales son esenciales para el cumplimiento de las metas para las que fueron creadas. Es así como su administración requiere medir la gestión, permitiendo emprender el mejoramiento de la institución. Por ende las nuevas maneras de gestionar se relacionan con la evaluación y control de los procesos.

De esta forma se tiene que los procesos administrativos según Robbins y Coulter (2013), bajo la premisa de funciones administrativas, lo definen de acuerdo con el esquema de las funciones de los gerentes que realizan ciertas actividades o deberes al tiempo que coordinan eficaz $y$ eficientemente el trabajo de los demás. De manera que indica que las funciones administrativas más importantes en las organizaciones son planear, organizar, dirigir y controlar.

Esta misma praxis es exigible en el caso de Latinoamérica. Fainstein (2009) afirma que en el ámbito organizacional se muestra una necesidad gerencial innovadora acorde a los tiempos en que se está viviendo, lo cual demanda desarrollar nuevos modelos culturales, con anticipación, innovación y solidaridad (rol social de las empresas y las organizaciones en general). De allí que el gerente educativo que promueva una gestión actualizada se ha convertido en una de las modas en la gestión de las organizaciones.

Señala que es así como, en la experiencia de América Latina en las últimas décadas, tanta historia de autoritarismo en conjunto con el albor democrático y una visión actual excesivamente material en las organizaciones, deviene en un capitalismo salvaje que dificulta que la gestión administrativa innovadora sea algo más que una moda. Por eso, como moda puede ser efímera. Pero, enfatiza que no hay otra forma de desarrollar un efectivo trabajo en equipo sino cambiando el concepto de moda por el de modo.

En este sentido, destaca que el tránsito hacia un modo de una gestión administrativa moderna no es fácil. Convertir los procesos administrativos en un modo de gestión organizacional requiere convicción, fijación de políticas y actitudes proactivas por parte de las personas que trabajan en la organización, lo cual no siempre sucede.

Destaca el mencionado autor que muchos fracasos de gestión, y en particular en los procesos de desarrollo de programas de calidad, grupos de calidad o mejora continua, son atribuidos por los propios responsables, a falencias o desatinos en tecnología gerencial para desarrollar y potenciar equipos de trabajo en sus organizaciones.

Aunado a lo anterior, es conveniente destacar que en efecto, se requiere de 
directores capacitados para dirigir la escuela como una organización social, tomando en cuenta las características particulares de la misma, lo cual necesita del mismo el ejecutar funciones de planificar, organizar, dirigir y controlar las actividades que ayuden a alcanzar las metas, como llevar a cabo el acompañamiento docente que permita evaluar el desempeño de este personal. De allí, se puede deducir que el gerente debe ser eficaz antes que eficiente, es decir no sólo hacer bien, sino sobre todo hacer lo que se considera correcto.

Sobre este parecer, Münch, Galicia, Jiménez y Pedronni (2011) definen la gestión escolar como la que "involucra la ejecución de las funciones de planificar, organizar, administrar personal, dirigir y controlar todos los aspectos y actividades de la organización educativa" (p 41). Además, necesita llevar a cabo los procesos organizacionales conformados por la toma de decisiones, el liderazgo y el acompañamiento al personal, en la gestión escolar, en lo pedagógico a los docentes, junto con las relaciones con la comunidad, ya que es determinante en el proceso enseñanza de sus estudiantes.

Así que los gerentes educativos necesitan estar dispuestos y conscientes de asumir la dirección de una institución, la cual no sólo se basa en obtener el perfil académico $\mathrm{y} / \mathrm{o}$ profesional sino también desarrollar la sensibilidad, humanidad, la proactividad y objetividad en el desempeño de sus labores, puesto que en la actualidad pueden observarse gerentes poco receptivos, autocráticos, los cuales se niegan a los cambios, reformas e innovaciones que se van desenvolviendo con las exigencias sociales, por ende, es necesario fomentar al máximo la productividad laboral, basado en la toma de decisiones acertadas para la organización y las personas que las integran.

Desde esta perspectiva, en Colombia esta forma de gestión escolar ha generado grandes transformaciones en las organizaciones educativas, donde la figura del director ha dejado de ser el eje central conductor de las actividades, encargado de trabajar con la transmisión de información y tramitación de documentos a ser un actor accesible, comunicador, generador de empatía y honestidad, cuyo perfil se encuentra dirigido a desarrollar conocimientos, experiencia y valores, conducentes a facilitar las acciones en su gestión, y por ende el liderazgo transformacional enmarcado en la efectividad, sin embargo, aparentemente estos desafíos no se están asumiendo a cabalidad.

Debido a que existen problemas de carácter administrativo pero además los cambios que afronta la gerencia colombiana, hacen necesario que las instituciones educativas cuenten con un nuevo perfil de gestión en sus procesos administrativos, la cual sea estratégica, visionaria, adaptada a los cambios, además, de saberlos interpretar, afrontarlos y generar las transformaciones necesarias, que garanticen resultados que brinden un clima laboral el cual favorezca a todos.

Siguiendo las ideas anteriores, es posible afirmar entonces, que el manejo administrativo se ha convertido en pilar del desarrollo de cualquier institución educativa, de tal manera, que el establecimiento y puesta en práctica de una excelente gestión 
administrativa no es sólo necesario, sino primordial, para promover un alto desarrollo organizacional. Los objetivos de la investigación fueron:

General: Analizar los procesos administrativos en la gestión escolar del director de las instituciones educativas oficiales del Departamento AtlánticoColombia.

Específicos: Describir el proceso de planeación en las instituciones educativas oficiales del Departamento AtlánticoColombia; caracterizar el proceso de organización en las instituciones educativas oficiales del Departamento AtlánticoColombia; describir el proceso de control en las instituciones educativas oficiales del Departamento Atlántico-Colombia; identificar las funciones de gestión escolar del director en las instituciones educativas oficiales del Departamento AtlánticoColombia; describir las habilidades del director en las instituciones educativas oficiales del Departamento AtlánticoColombia.

En el marco de los objetivos antes mencionados se destacó en la investigación la variable Procesos Administrativos, pues conceptualmente como un aspecto fundamental como son los procesos administrativos, los cuales pueden definirse según Stoner (2012) un proceso administrativo es una forma sistemática de hacer las cosas. Se habla de la administración en términos de tres funciones específicas de los gerentes: la planificación, la organización, y el control.

En otras palabras, la administración es el proceso de planificar, organizar y controlar las actividades de los miembros de la organización, es decir, de las instituciones educativas y el empleo de los demás recursos organizacionales, con el propósito de alcanzar las metas establecidas en la organización de una manera adecuada.

Por otra parte, Münch (2013) define el proceso como un conjunto de pasos o etapas necesarias para llevar a cabo una actividad. En su concepción más sencilla se puede definir el proceso administrativo como la administración en acción, o también como el conjunto de etapas sucesivas a través de las cuales se efectúa la administración, mismas que se interrelacionan y forman un proceso integral.

Quiere decir entonces, que a partir de lo referido por este autor el proceso administrativo viene dado por el cumplimiento de una serie de etapas, las cuales son necesarias para el cumplimiento o desarrollo adecuado de una actividad, que se interrelacionan para así alcanzar los objetivos establecidos con anterioridad dentro de la organización.

Así mismo Robbins y Coulter (2013), bajo la premisa de funciones administrativas, lo definen de acuerdo con el esquema de las funciones de los gerentes que realizan ciertas actividades o deberes al tiempo que coordinan eficaz y eficientemente el trabajo de los demás. De manera que indica que las funciones administrativas más importantes en las organizaciones son planear, organizar, dirigir y controlar.

El proceso administrativo es un pilar fundamental de la organización, es la forma en que se organiza el trabajo y se distribuyen las tareas para focalizar las operaciones orientándolas al alcance de metas, tomando en cuenta a todo el 
personal, así como también todos aquellos recursos necesarios para tal fin, en tal sentido se fija posición con el autor Stoner (2012).

\section{MÉTODO}

En esta sección se exponen los aspectos metodológicos, técnicas, instrumentos, así como también los procedimientos que fueron empleados para la recolección y procesamiento de los datos, además se describe el tipo de estudio, de manera que se extraigan de la realidad seleccionada lo necesario para análisis de los procesos administrativos en la gestión escolar del director de las instituciones educativas oficiales del Departamento AtlánticoColombia, la investigación se consideró de tipo descriptivo ya que se estudiaron las variables limitándose a su descripción. El diseño de investigación debe tener en cuenta que este diseño describe las variables procesos administrativos en la gestión escolar del director, en una población conformada por el personal de las instituciones educativas oficiales del Departamento Atlántico-Colombia.

Por otro lado, es transversal o transeccional, por cuanto se recogió la información en un momento único del proceso, para describirlas, analizando su incidencia o interrelación en un momento dado. La población estuvo conformada por las instituciones educativas oficiales del Departamento Atlántico-Colombia, organizadas de la siguiente manera:

Tabla 1. Población en Estudio

\begin{tabular}{lccc}
\hline INSTITUCIÓN EDUCATIVA & DIRECTIVOS & DOCENTES & TOTAL \\
\hline I.E Normal superior de Manatí & 2 & 23 & 25 \\
I.E San Luis Beltrán & 3 & 19 & 21 \\
I.E Juan Bosco & 2 & 32 & 34 \\
I.E De Sabana Larga & 1 & 72 & 73 \\
I.E Nuestra señora del tránsito & 3 & 32 & 35 \\
I.E Santa Lucia & 2 & 29 & 31 \\
Politécnico de Soledad Departamento & 2 & 60 & 62 \\
del Atlántico & & & \\
I.E De Bohórquez & 1 & 26 & 27 \\
\hline
\end{tabular}

Fuente: Nómina de los planteles (2016)

La Muestra para efectos de este trabajo de investigación se tomó de un diseño no probabilístico ya que en este caso la elección de los elementos no depende de la probabilidad, sino de causas relacionadas con las características de la investigación o de quien hace la muestra. Aquí el procedimiento no es mecánico ni con base en fórmulas de probabilidad, sino que depende del proceso de toma de decisiones de un investigador $\mathrm{o}$ de un grupo de investigadores $\mathrm{y}$, desde luego, las muestras seleccionadas obedecen a otros criterios de investigación. Partiendo de lo anterior, para seleccionar la muestra, se tuvo en cuenta que presenta la problemática planteada en el primer capítulo, además de otros criterios como la facilidad de recogida de 
información, excelente ubicación geográfica, que la institución cuente con más de cuatro directivos y más de cuarenta docentes, que posee un alto índice de matrículas en el Departamento Atlántico-Colombia y alrededores, como institución tienen una trayectoria de funcionamiento, este es el caso de las I.E De Sabana Larga y la I.E Nuestra Señora del Tránsito.

Es por ello que para esta investigación en particular la muestra quedó constituida por ciento ocho sujetos, específicamente cuatro directivos y ciento cuatro docentes, siendo estos últimos una muestra control, que prestan sus servicios profesionales en el centro educativo teniendo en cuenta sus características, roles, desempeños y relaciones desarrolladas en el contexto escolar, tal y como se muestra en la siguiente Tabla:

Tabla 2. Muestra de la Población

\begin{tabular}{lccr}
\hline INSTITUCIÓN EDUCATIVA & DIRECTIVOS & DOCENTES & TOTAL \\
\hline I.E De Sabana Larga & 1 & 72 & 73 \\
I.E Nuestra señora del tránsito & 3 & 32 & 35 \\
\hline
\end{tabular}

Fuente: Nómina del Plantel (2016)

La técnica de Recolección de Datos resultó imprescindible para llevar a cabo este proceso de investigación, que se diseñen unas líneas de orientación metodológicas, para guiar las acciones y ejecutar la recolección de datos propios del objeto de estudio de esta investigación. Así entonces, con el propósito de darle cumplimiento a la misma, se consideró conveniente la utilización de técnicas de recolección de datos cuantitativas para medir las variables de estudio.

Así, en este caso se utilizó la observación mediante encuesta, la cual es definida por Sabino (2010), como la obtención de los datos de interés en la investigación mediante la interrogación a los miembros del universo en estudio. De igual manera, el instrumento básico de la observación mediante encuesta es el cuestionario, el cual se define de acuerdo al autor mencionado, como un conjunto de preguntas, preparado sobre los hechos y aspectos que interesan en una investigación, para su contestación por la población o muestra a la que se extiende el estudio emprendido; bajo tales perspectivas, en la presente investigación se utilizó el cuestionario simple.

De acuerdo a los planteamientos realizados en el segmento anterior, el instrumento consta de cuarenta y ocho (48) ítems, conformados por afirmaciones con selección de respuesta en escala tipo Likert, cuyas ponderaciones van del 1 al 4, siendo las opciones de respuesta: nunca, casi nunca, casi siempre y siempre, respectivamente. A tales opciones, se aplicaran sus respectivos puntajes con respecto a las afirmaciones, las cuales se describen en el siguiente cuadro: 
Tabla 3. Ponderación de Opciones de Respuesta

\begin{tabular}{cc}
\hline Afirmación & Opción de Respuesta \\
\hline 4 & Siempre \\
3 & Casi siempre \\
2 & Casi nunca \\
1 & Nunca \\
\hline
\end{tabular}

Fuente: Ortega (2017)

La validez y confiabilidad del Instrumento de recolección de datos se determina con exactitud lo que efectivamente se pretende medir en una investigación; de allí que la aplicación de un instrumento al momento de recabar información útil para el desarrollo de un trabajo o estudio de investigación conlleva necesariamente a validar su contenido, como lo expresan Hernández y col. (2010). En este sentido el instrumento de medición se validó tomando en consideración la opinión de expertos en el área. Para cumplir con esta actividad se sometió el instrumento, a un proceso de validación de contenido, metodología, a través del juicio de cinco expertos del área de educación y metodología, quienes evaluaron cada interrogante planteada y calificaron el ajuste de las mismas para medir la variable procesos administrativos en la gestión escolar del director.
Una vez realizada la prueba piloto, se procedió a tabular los datos, luego se sustituyeron los valores de las respectivas variables de la fórmula antes planteada, finalmente, al aplicar la fórmula Alfa de Cronbach, se obtuvo el como resultado el coeficiente $r_{t t}=0,95$, considerando en tal sentido, que el instrumento diseñado por la investigadora del presente estudio tiene una confiabilidad Muy Alta.

En este estudio, para la codificación, tabulación, análisis de los datos se utilizó la estadística descriptiva, como lo señalan Hernández y col. (2010), indicando la frecuencia en las respuestas de los encuestados, ubicando los promedios dados de acuerdo a la mayor tendencia, mostrando también la media aritmética para categorizar los resultados con base en el baremo diseñado por la investigadora.

La categorización de los valores presentados con la calificación, se realizan el siguiente baremo:

Tabla 4. Baremo

\begin{tabular}{ll}
\hline Escala de Valores & Alternativas \\
\hline $1.00---1.75$ & Nunca \\
$1.76---2.50$ & Casi Nunca \\
$2.51-----3.25$ & Casi Siempre \\
$3.26----4.00$ & Siempre \\
\hline
\end{tabular}

Fuente: Ortega (2017) 
RESULTADOS

Se expone esta sección del artículo por dimensiones.

Dimensión Proceso de Planeación: Para esta importante fase de los procesos administrativos la Tabla 5 y gráfico 3 registran un resultado altamente favorable:
$100 \%$ de respuestas de las categorías Siempre y Casi Siempre. Atendiendo a este hallazgo, el personal directivo consideró que en su acción gerencial piensan con antelación en sus metas y acciones, dado que basan sus actos en algún método, plan o lógica, y no en corazonadas.

Tabla 5. Dimensión Proceso de Planeación

\begin{tabular}{clcc}
\hline Categorías & Frecuencia & Porcentaje & Porcentaje acumulado \\
\hline 4,00 & 2 & 50,0 & 50,0 \\
5,00 & 2 & 50,0 & 100,0 \\
Total & 4 & 100,0 & \\
\hline
\end{tabular}

Fuente: SPSS aplicado a la base de datos (2017)

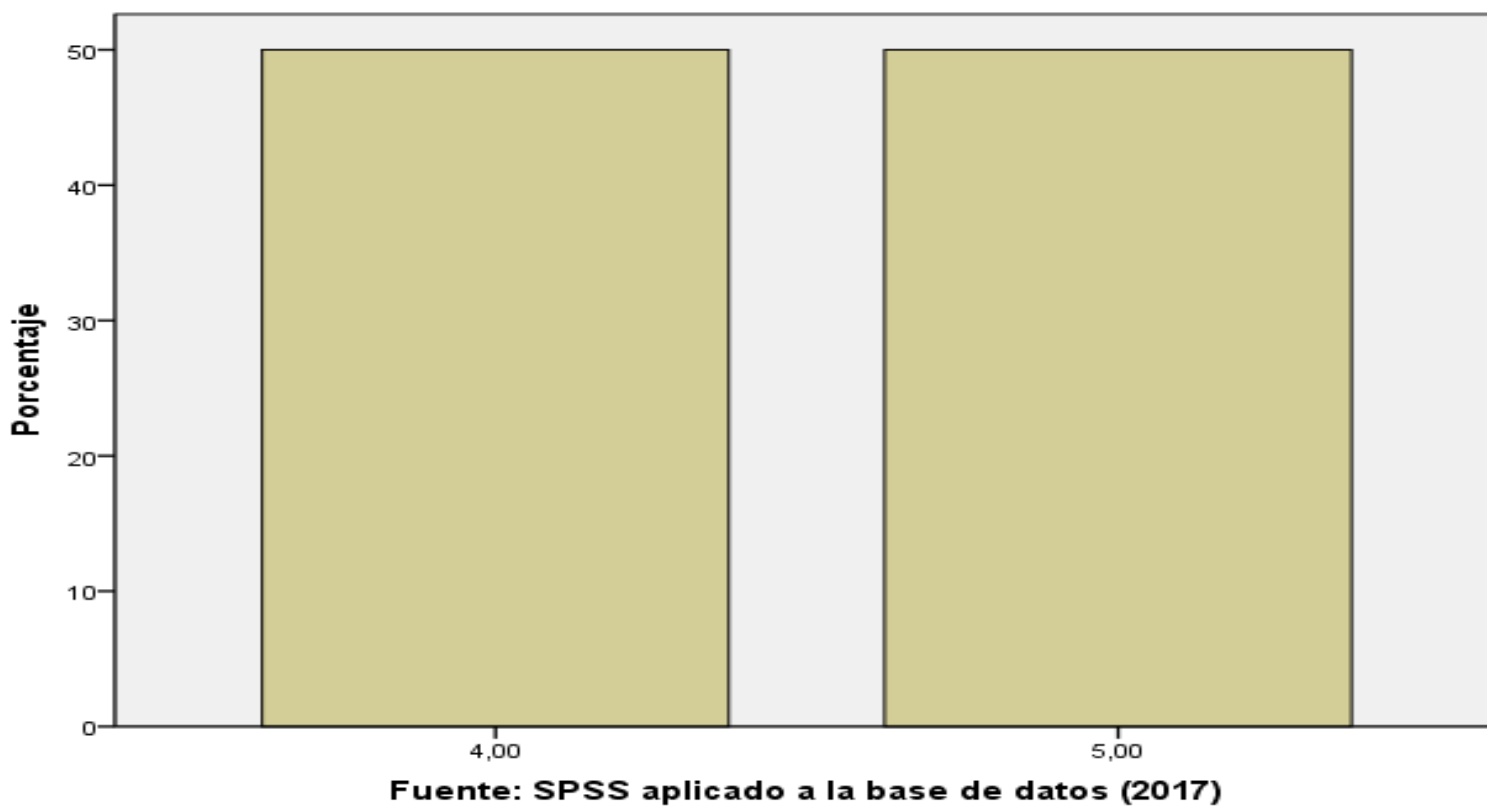

Gráfico 1. Dimensión Proceso de Planeación.

Más aún, los planes representan los objetivos de la organización así como también establecen los procedimientos idóneos para alcanzarlos. Sin embargo, al contrastar esta autoevaluación de los directivos con la percepción que tienen los docentes de su gestión, se encontró que se encuentran en franca oposición.

Dimensión Proceso de Organización: el hallazgo que se obtuvo en este rubro reitera la tendencia altamente positiva: 100\% de respuestas favorables. 
Tabla 6. Dimensión Proceso de Organización

\begin{tabular}{cllc}
\hline Categorías & Frecuencia & Porcentaje & Porcentaje acumulado \\
\hline 4,00 & 1 & 25,0 & 25,0 \\
5,00 & 3 & 75,0 & 100,0 \\
Total & 4 & 100,0 & \\
\hline
\end{tabular}

Fuente: SPSS aplicado a la base de datos (2017)

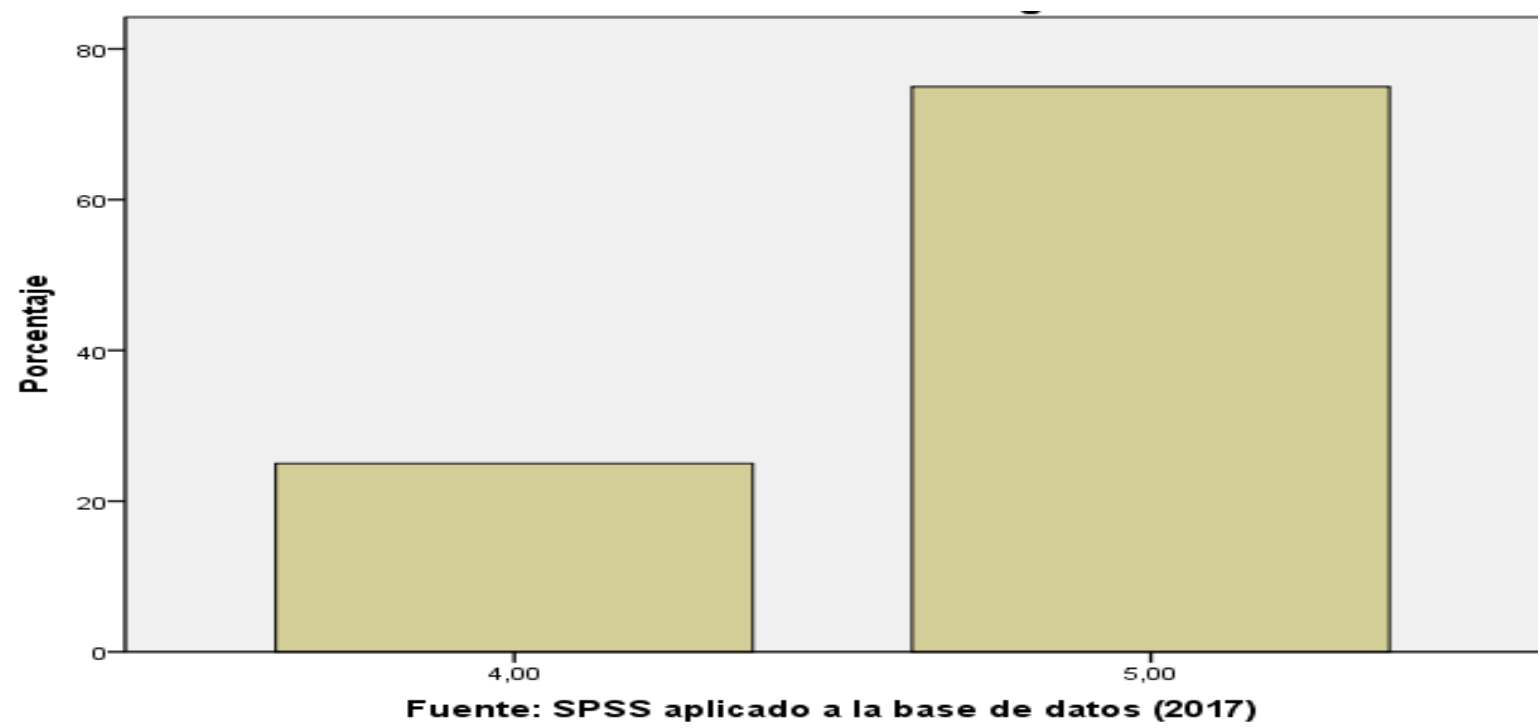

Gráfico 2. Dimensión Proceso de Organización

Este valor puede tomarse como evidencia para afirmar que, según la opinión del plantel directivo, la acción de los gerentes educativos de las instituciones investigadas desarrolla procesos en los cuales se ordena, distribuye el trabajo, la autoridad y los recursos entre los integrantes de la organización para alcanzar las metas establecidas. Pero esta valoración es completamente contraria al juicio poco favorable que en este aspecto tienen los docentes de la gerencia de las instituciones investigadas.

Dimensión Proceso de Control: En esta dimensión los resultados que muestran la Tabla 7 y gráfico 3 hablan por sí solos:

Tabla 7. Dimensión Proceso de Control

\begin{tabular}{cccc}
\hline Categorías & Frecuencia & Porcentaje & Porcentaje acumulado \\
\hline 5,00 & 4 & 100,0 & 100,0 \\
\hline
\end{tabular}

Fuente: SPSS aplicado a la base de datos (2017) 


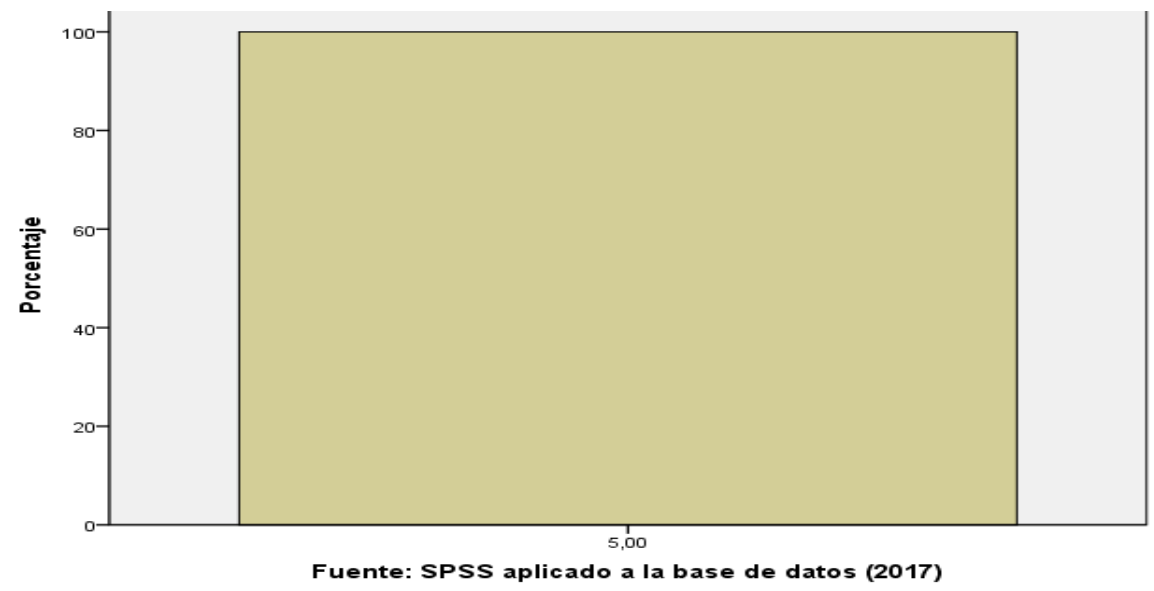

Gráfico 3. Dimensión Proceso de Control

La totalidad de los directivos tienen un juicio favorable de su gestión en este renglón. Atendiendo a este valor, el plantel directivo establece sistemas para medir los resultados y corregir las desviaciones que se presenten, con el fin de asegurar que los objetivos y planes planteados se logren. Pero aquí nuevamente se refleja la franca contradicción con la valoración que hacen los docentes de la gerencia institucional.

Dimensión Funciones de Gestión Escolar La Tabla 8 y gráfico 4 evidencian un resultado por demás positivo: $100 \%$ de respuestas consideradas como favorables (categoría Siempre)

Tabla 8. Dimensión Funciones de Gestión Escolar

\begin{tabular}{cccc}
\hline Categorías & Frecuencia & Porcentaje & Porcentaje acumulado \\
\hline 5,00 & 4 & 100,0 & 100,0 \\
\hline
\end{tabular}

Fuente: SPSS aplicado a la base de datos (2017)

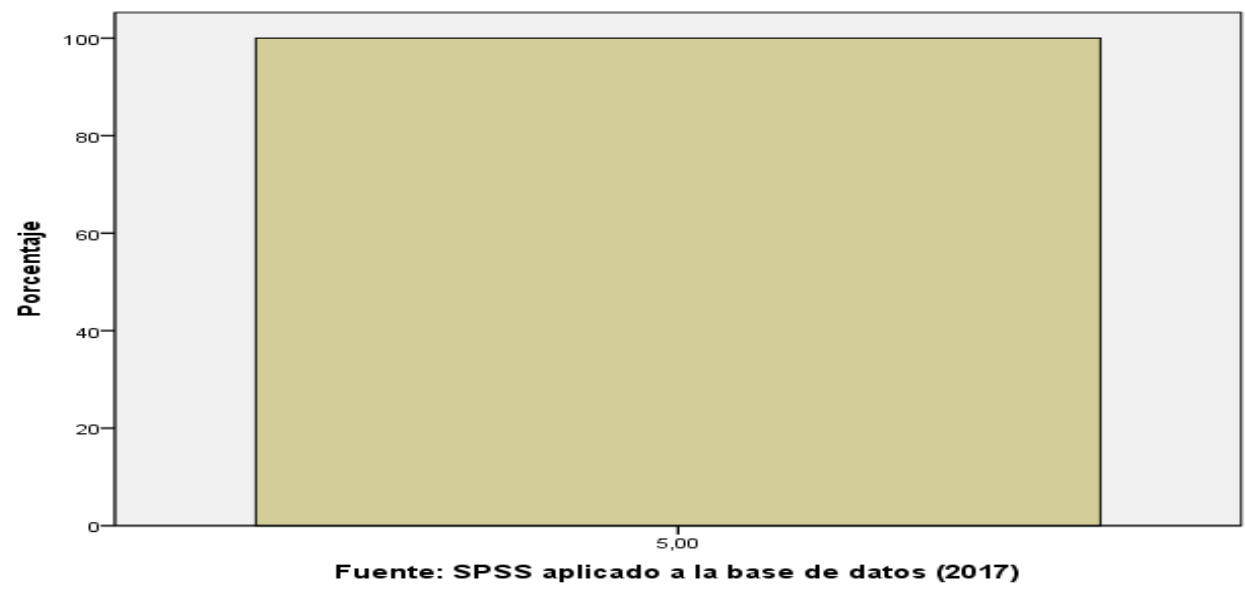

Gráfico 4: Dimensión Funciones de Gestión Escolar

Este valor indica que atendiendo a la percepción del plantel directivo, la gerencia 
de las instituciones investigadas ejerce las funciones pedagógicas, administrativas, autogestión de recursos y relaciones con la comunidad con la finalidad de convertirse en pieza clave para lograr la marcha de las acciones, evidenciando una institución con integrantes preocupados por obtener una educación de calidad. Los hallazgos se corresponden con los referentes teóricos de López (2012), quien indica que los directores son gestores intermedios que deciden los asuntos del plantel y en la gestión escolar ejercen funciones precisas de planificar, organizar, administrar personal, dirigir y controlar las acciones mediante procesos organizacionales; además, de la toma de decisiones, el liderazgo y el acompañamiento pedagógico coherente con el ideario de la educación.

La confrontación que se tiene con el juicio docente sobre el desempeño de los gerentes educativos es reiterativa, al calificar desfavorablemente su gestión.

Tabla 9. Dimensión Habilidades

\begin{tabular}{cccc}
\hline Categorías & Frecuencia & Porcentaje & Porcentaje acumulado \\
\hline 5,00 & 4 & 100,0 & 100,0 \\
\hline
\end{tabular}

Fuente: SPSS aplicado a la base de datos (2017)

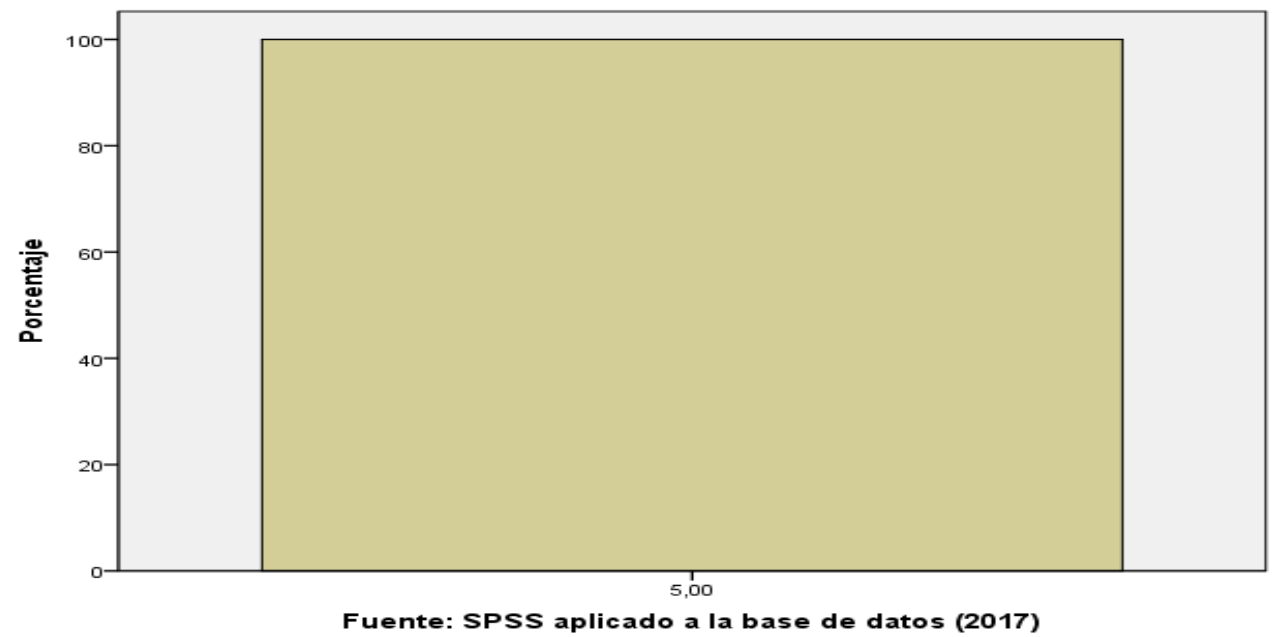

Gráfico 5. Dimensión Habilidades

Los resultados arrojados en la Tabla 9 y gráfico 5 evidencian la tendencia positiva en esta dimensión, puesto que se obtiene el $100 \%$ de respuestas favorables. Esta autovaloración hecha por los directivos indica que, en su opinión, poseen y aplican habilidades adecuadas a las exigencias actuales del sistema educativo basado en las estrategias participativas para utilizarlas en su acción y así orientar correctamente a los docentes, utilizando para ello métodos y técnicas que exijan la corresponsabilidad activa de los mismos, desarrollando relaciones personales que fortalezcan excelentes actitudes e ideales. Sin embargo, estos juicios favorables contrarían las apreciaciones hechas por el personal docente sobre la labor que desempeña el 
personal directivo de las instituciones investigadas. En sentido, los hallazgos van en concordancia con la teoría de Mosley, Megginson y Pietri (2009), quienes afirman que los directivos de las instituciones educativas deben mostrar durante su actuación como director habilidades conceptuales, habilidades para relacionarse con las personas a su cargo y habilidades técnicas, como complemento a todas las áreas afines a su especialidad.

Los resultados para la variable la Tabla 10 y gráfico 6 exhibe una derivación altamente favorable: $100 \%$ de respuestas de la categoría Siempre. Este valor puede tomarse como elemento de convicción para afirmar que, en la autoevaluación de la gestión del personal directivo, su desempeño gerencial desarrolla los procesos administrativos dentro de las instituciones educativas que vienen dados por la interacción y el trabajo en equipo entre sus miembros, para de esta manera llevar a cabo la planeación, organización y control, en todos y cada uno de los proyectos educativos a ejecutar, cumpliendo así con la misión y visión de la organización.

Tabla 10. Variable Procesos Administrativos en la Gestión Escolar del Director

\begin{tabular}{cccc}
\hline Categorías & Frecuencia & Porcentaje & Porcentaje acumulado \\
\hline 5,00 & 4 & 100,0 & 100,0 \\
\hline
\end{tabular}

Fuente: SPSS aplicado a la base de datos (2017)

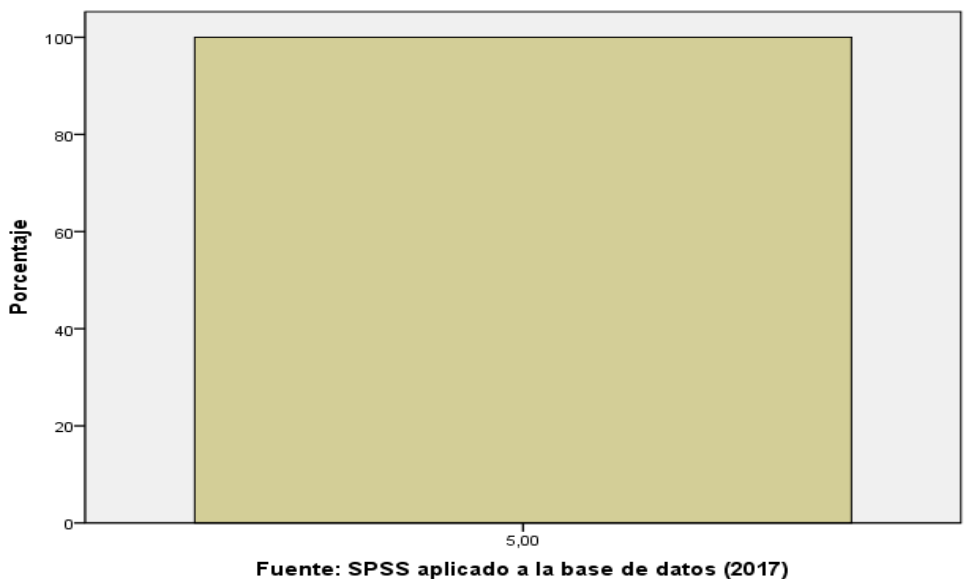

Gráfico 6. Variable Procesos Administrativos en la Gestión Escolar del Director

Los resultados se corresponden con los referentes teóricos de López (2012), quien indica que los directores son gestores intermedios que deciden los asuntos del plantel y en la gestión escolar ejercen funciones precisas de planificar, organizar, administrar personal, dirigir y controlar las acciones mediante procesos organizacionales; además, de la toma de decisiones, el liderazgo y el acompañamiento pedagógico coherente con el ideario de la educación. Aquí nuevamente esta percepción de los directivos contradice abiertamente el juicio que hacen los docentes de las instituciones investigadas con respecto al desempeño gerencial de aquellos. 


\section{CONCLUSIONES}

En relación al primer objetivo específico: Describir el proceso de planeación en las instituciones educativas oficiales del Departamento Atlántico-Colombia. Al respecto se tiene que los directivos de estas instituciones, expresaron que siempre refieren el proceso de planeación administrativa en las instituciones educativas en contexto. Sin embargo, los docentes afirmaron que los directivos casi nunca aplican el proceso de planeación para establecer la misión, visión, objetivos y metas de la institución educativa, observándose una marcada diferencia entre sus respuestas.

Tal afirmación se deriva de la manifestación de los docentes en cuanto a que los directivos no fundamentan la planificación de las actividades en función de la misión de la institución, ni describen detalladamente la razón de ser de la comunidad educativa; así como, tampoco identifican las competencias educativas de la institución a través de la misión.

Además, no consideran que la visión de la institución es realista en lo que quiere llegar a ser; así como, no tienen una idea clara del futuro organizacional de la comunidad educativa, lo que dificulta poder medir la visión de la institución en el tiempo. De igual manera, los directivos no establecen los resultados globales que la organización espera alcanzar, mediante la definición de los objetivos de la institución educativa a ser logrados en un tiempo determinado, ni tampoco fijan objetivos concretos de desempeño para todos los miembros de la institución. Así mismo, los directivos casi nunca fijan las metas por cada departamento de la institución, ni establecen programas para alcanzarlas de una forma sistemática, ni marcan la dirección de las decisiones administrativas.

En relación al segundo objetivo específico: Caracterizar el proceso de organización en las instituciones educativas oficiales del Departamento AtlánticoColombia. Al respecto se tiene, que los directivos de estas instituciones, respondieron que casi siempre caracterizan el proceso de organización en las instituciones educativas en contexto. Sin embargo, los docentes afirmaron que los directivos casi nunca tienen presente el proceso de organización para definir la estructura organizacional, la especialización del trabajo y la departamentalización de la institución educativa, observándose discrepancia entre sus respuestas.

Esta discrepancia se debe, según los docentes, a que los directivos casi nunca diseñan una estructura organizacional que facilite el trabajo a los integrantes de la institución, con la finalidad de distribuir de manera flexible las funciones dentro de la misma para lograr el éxito y coordinar las actividades educativas equilibradamente. Además, casi nunca los directivos asignan a cada miembro una tarea específica en el desarrollo de los programas educativos, ni utilizan la especialización del trabajo como un mecanismo de organización; así como tampoco, consideran que cada miembro deba ser experto en el área donde se desenvuelve.

De igual manera, casi nunca los directivos agrupan los trabajos dentro de la institución de acuerdo a las necesidades de la misma; ni crean equipos de trabajos para 
que cumplan con funciones similares y tampoco consideran que los miembros de un mismo departamento compartan necesidades comunes.

En relación al tercer objetivo específico: Describir el proceso de control en las instituciones educativas oficiales del Departamento Atlántico-Colombia. Al respecto se tiene, que los directivos de estas instituciones, respondieron de forma dividida que siempre y casi siempre, describen el proceso de control en las instituciones en contexto. Sin embargo, los docentes afirmaron que los directivos casi nunca aplican el proceso de control para la identificación del estándar, ni para establecer medidas correctivas; observándose así, divergencia entre las respuestas de los directivos y docentes.

En este sentido, la divergencia se debe según los docentes, a que casi nunca los directivos establecen controles a través de una medición estándar, dejando así de comparar los resultados reales con los propuestos en cada actividad educativa; ni verifican los resultados obtenidos para conocer si lo obtenido fue lo realmente planificado.

De igual manera, los docentes afirmaron que casi nunca los directivos rediseñan los procesos administrativos de la institución que permitan la identificación a tiempo de los factores que inciden en el desempeño laboral de los miembros de la comunidad educativa, ni ofrecen motivación de manera constante al personal de la institución para alzar exitosamente los objetivos.

En relación al cuarto objetivo específico: Identificar las funciones de gestión escolar del director en las instituciones educativas oficiales del Departamento AtlánticoColombia. Al respecto se tiene, que los directivos de estas instituciones, respondieron que siempre identifican las funciones de gestión escolar del director en las instituciones en contexto. Sin embargo, los docentes afirmaron que los directivos casi nunca utilizan la función pedagógica, administrativas, autogestión de curso y relaciones con la comunidad en las instituciones educativas; observándose así, diferencia entre las respuestas de los directivos y docentes.

En este orden de ideas, tal afirmación es debido a que los docentes consideraron que los directivos casi nunca hacen adecuaciones al currículo para adaptarla a las características de los estudiantes, ni promueven la calidad en la práctica pedagógica en el aula del centro educativo y tampoco buscan formar a un ciudadano crítico desde los procesos pedagógicos que se gestan en la escuela.

De igual manera, los docentes expresaron que los directivos casi nunca promueven la unificación entre las prácticas pedagógicas con los recursos con que se cuentan, ni desarrollan acciones para garantizar el alcance de los objetivos desde la participación de todos en los ámbitos que conforman la escuela y tampoco optimizan de manera integrada los recursos que dirigen para el logro de la formación profesional de su personal. Además, casi nunca se toman el tiempo necesario en la toma de decisiones en función de cada actividad a desarrollarse para la autogestión de los recursos, así como tampoco utilizan racionalmente los recursos dedicados a las actividades de la institución educativa. 
Así mismo, los docentes afirmaron que los directivos casi nunca socializan el Proyecto Educativo de la escuela integrando a la comunidad, no vinculan el aprendizaje escolar con el entorno comunitario; así como tampoco, promueven acciones para impulsar el desarrollo de la comunidad donde está ubicada la escuela.

En relación al quinto objetivo específico: Describir las habilidades del director en las instituciones educativas oficiales del Departamento Atlántico-Colombia. Al respecto se tiene, que los directivos de estas instituciones, respondieron que siempre describen las habilidades del director como componente de la gestión escolar en contexto. Sin embargo, los docentes afirmaron que los directivos casi nunca tienen presente las habilidades conceptuales, relaciones personales y habilidades técnicas como parte de su gestión escolar en las instituciones educativas, observándose de esta manera, diferencias entre las respuestas de los directivos y docentes.

En este sentido, tal diferencia se debe, según los docentes, a que los directivos casi nunca resuelven los problemas en beneficio de todas las personas implicadas, ni poseen destrezas de razonamiento lógico como elementos de predicción sobre la eficacia administrativa y tampoco asumen la importancia de la función que realiza. Además, los directivos casi nunca manifiestan confianza hacia el personal, es irrespetuoso de las necesidades de otras personas, ni tolerante con el trabajo en grupo. Así como tampoco, conocen los aspectos técnicos de su trabajo, impidiéndole saber guiar a los demás, tampoco poseen la capacidad para usar el conocimiento como destreza especial para realizar determinadas tareas.

En cuanto al sexto objetivo específico: Formular lineamientos teórico-prácticos sobre los procesos administrativos en la gestión escolar del director de las instituciones educativas oficiales del Departamento Atlántico-Colombia. Se formularon lineamientos teóricos prácticos que podrán contribuir a mejorar los procesos administrativos en la gestión escolar de las instituciones en contexto; así como, ampliar las competencias de directivos y docentes en esta materia.

Finalmente, en cuanto al objetivo general: Analizar los procesos administrativos en la gestión escolar del director de las instituciones educativas oficiales del Departamento AtlánticoColombia, se puede concluir, que las instituciones educativas en contexto, se detectaron debilidades por parte de los directivos, de acuerdo a la percepción de los docentes, en cada una de las dimensiones de las variables objetos de estudio. Por lo que representa una posible intervención de las autoridades de la comunidad educativa.

\section{REFERENCIAS}

Fainstein, J (2009) La Toma de Decisiones de la Empresa. Buenos Aires: Ed. Atlántida Hernández, Fernández y Baptista (2010). Metodología de la investigación. México D.F: Editorial. Mc Graw-Hill

López, M. (2012). Investigación en administración en América Latina. Recuperado de http://books.google.co.ve/books?id=N1/ 2My4B7h8C\&pg=PA129\&lpg =PA129\&d $q=E s+u n+$ conjunto + de+ideas+generale $\mathrm{s}$, +algunas+de+ellas+abstractas.

Consultado en 18-07-2017 
López, R. (2012). Manual del Supervisor, Director y Docente. Volumen 5. Caracas: Publicaciones Monfort. C.A

Mosley, D., Megginson, L. y Pietri, P. (2009). Supervisor y la Práctica del Empawerment. México: Grupo GEO. Impresores

Münch, L., Galicia, E., Jiménez, S. y Pedronni, F. (2011). Administración de Instituciones Educativa. México: Editorial Trillas

Münch, L y García M, (2013). Fundamentos de Administración, 2a ${ }^{a}$. ed., Editorial: Trillas, México
Münch, L. (2009). Fundamentos de la Administración. México. Editorial Trillas. Ministerio de Educación y Deportes

Robbins, S. y Coulter, M. (2013). Administración. 6ta. Edición. México: Prentice Hall Hispanoamericana, S.A

Sabino, C (2010). El proceso de investigación. México: Editorial Panapo de Venezuela y Hall Hispanoamericana 